\title{
Cooperativity Effects on the Hydrogen Bonds Within HCN and HNC Complexes
}

\author{
Paulo M. C. de Oliveira, Juliana A. B. Silva \& Ricardo L. Longo
}

\section{Introduction}

Hydrogen bonding (H-bond) remains relevant because of its importance in Chemistry, Biology and Materials Science. The diversity and peculiarities of H-bonds still cause debates regarding, for instance, the behavior and properties simple liquids such methanol and water. Another aspect is the relationships between the topological and statistical mechanics descriptors of complex H-bond networks and the properties of liquids and mixtures. ${ }^{1-3}$ For instance, the presence of small-world patterns in H-bond networks of aqueous mixtures may be related to special behavior of these networks, such as resilience and rapid response to perturbation. ${ }^{1-3}$ These characteristics may be helpful to explain and interpret various physical chemistry properties of aqueous solutions. Thus, it is important to extend these statistical mechanics analyses to more diverse H-bond networks, such as pure HCN and HNC liquids and their mixtures. The H-bond networks used in these analyses are obtained from computer simulations employing classical intermolecular interaction potentials, usually obtained by parametrization from the liquid experimental properties. However, these potentials are not available for $\mathrm{HCN} / \mathrm{HNC}$ systems, which leads to their parametrization with respect to high-level quantum chemical calculations or to quantum computer simulations such as the Born-Oppenheimer Molecular Dynamics (BOMD) method.

$\mathrm{HCN}$ was chosen because it is a polar compound that may form H-bond networks containing linear, branched and cyclic aggregates. ${ }^{4,5}$ In addition, $\mathrm{HCN}$ may be a relevant prebiotic and extraterrestrial species related to the origin of life. 6 Thus, the topological properties of the H-bond bond networks in liquid $\mathrm{HCN}$ and $\mathrm{HCN}$ HNC mixtures could be relevant in understanding their thermodynamics and structural properties. In addition, it has shown that the H-bond cooperativity effects are important for several properties. ${ }^{7-9}$ Computer simulation methods such as Monte Carlo and molecular dynamics do capture many-body effects on the H-bonds. However, these H-bond cooperativity effects require specialized intermolecular potentials or the use of quantum methods that describe these effects properly.

Thus, the quantitative properties of the H-bonds in $\mathrm{HCN}-\mathrm{HNC}$ dimeric and trimeric complexes are investigated in detail by electronic structure methods. This aims at providing a benchmark for selecting densityfunctionals to be employed in BOMD simulations or a database for parametrization of the intermolecular interaction potentials for $\mathrm{HCN} / \mathrm{HNC}$ systems.

\section{Methods}

All possible dimeric and trimeric $\mathrm{HCN}-\mathrm{HNC}$ complexes were fully optimized with the MP2/6$311++\mathrm{G}(\mathrm{d}, \mathrm{p})$ method without symmetry constraints. Energy (single-point) calculations were performed with global-hybrid (B3LYP, BP86 and PBE) and metaGGA (TPSS) density functionals, with long-range and 
Table 1. Interaction energies $\left(\mathrm{kJ} \mathrm{mol}^{-1}\right)$ calculated for a set of density functionals, with dispersion (GD3) and long-range (LR) corrections, using the ccpVDZ basis set and BSSE corrected values are in parenthesis.

\begin{tabular}{|c|c|c|c|c|}
\hline & HCN-HCN & HCN-HNC & HNC-HCN & HNC-HNC \\
\hline B3LYP & $21.63(16.69)$ & $31.71(25.48)$ & $20.92(17.74)$ & $33.14(28.49)$ \\
\hline B3LYP-GD3 & $24.64(19.71)$ & $35.27(29.00)$ & $23.05(19.87)$ & $36.69(32.05)$ \\
\hline B3LYP-LR & $24.10(18.95)$ & $34.85(28.28)$ & $22.80(19.41)$ & $35.40(30.46)$ \\
\hline TPSS & $20.59(16.36)$ & $31.13(25.73)$ & $20.59(18.12)$ & $33.60(29.96)$ \\
\hline TPSS-GD3 & $23.60(19.37)$ & $34.64(29.25)$ & $22.72(20.25)$ & $37.15(33.51)$ \\
\hline TPSS-LR & $23.97(19.79)$ & $34.52(29.12)$ & $22.13(19.66)$ & $34.02(30.33)$ \\
\hline BP86 & $19.33(14.56)$ & $29.87(23.85)$ & $19.62(16.57)$ & $32.68(28.28)$ \\
\hline BP86-GD3 & $22.34(17.57)$ & $33.39(27.41)$ & $21.76(18.70)$ & $36.23(31.88)$ \\
\hline BP86-LR & $26.53(22.01)$ & $38.16(32.34)$ & $24.77(21.92)$ & $37.82(33.68)$ \\
\hline PBE & $22.72(17.61)$ & $33.56(27.15)$ & $23.05(19.75)$ & $36.61(31.92)$ \\
\hline PBE-GD3 & $25.73(20.63)$ & $37.07(30.71)$ & $25.15(21.84)$ & $40.17(35.48)$ \\
\hline PBE-LR & $24.60(20.21)$ & $35.61(29.96)$ & $22.89(20.21)$ & $35.35(31.42)$ \\
\hline CCSD(T)/CBS & 19.76 & 29.92 & 19.93 & 30.98 \\
\hline
\end{tabular}

dispersion corrections. The following basis sets were employed: cc-pVXZ with $\mathrm{X}=\mathrm{D}, \mathrm{T}$ and $\mathrm{Q}$. The basisset superposition error (BSSE) in the interaction energies were corrected using the counterpoise method.

As reference, the H-bond energies were calculated with the ab initio correlated $\operatorname{CCSD}(\mathrm{T})$ method extrapolated to the complete basis-set (CBS) limit. This extrapolation was performed by applying the Eqs. 1 and 2 to evaluate the corresponding correlation (corr) and Hartree-Fock (HF) energies: ${ }^{10,11}$

$$
\begin{aligned}
& E^{c o r r}(X)=E^{c o r r}(\infty)+A^{\text {corr }} X^{-\alpha} \\
& E^{H F}(X)=E^{H F}(\infty)+A^{H F} X^{-\beta}
\end{aligned}
$$

where $X=2$ for the cc-pVDZ basis and $X=3$ for the cc$\mathrm{pVTZ}$ basis set, and $\alpha$ and $\beta$ are optimized exponents. 10,11

\section{Results and Discussion}

The reported experimental binding energy for the HCN-HCN dimer the gas-phase is $15.9 \pm 0.67 \mathrm{~kJ}$ $\mathrm{mol}^{-1} .^{12}$ There is also an older reported value of 10.88 $\mathrm{kJ} \mathrm{mol}^{-1}$ obtained from vapor density data. ${ }^{13}$ The binding energy calculated in this work for $\operatorname{CCSD}(\mathrm{T}) / \mathrm{CBS}$ for $\mathrm{HCN}$ dimer is $19.8 \mathrm{~kJ} \mathrm{~mol}^{-1}$. Employing the zero-point vibrational energy (ZPE) contribution $(5.1 \mathrm{~kJ}$ mol-1) calculated with the MP2/cc-pVTZ method, provides a corrected ZPE value $\left(14.7 \mathrm{~kJ} \mathrm{~mol}^{-1}\right)$ in excellent agreement with the experiment based on spectroscopic data. ${ }^{12}$ The binding energies calculated for the HCN/ HNC dimers are summarized on Table 1 and consider the BSSE, dispersion and long-range contributions separately employing density-functionals available in the CP2K program.

Regardless of the method or the corrections employed, the H-bonds with $\mathrm{HNC}$ as donor (e.g., $\mathrm{HCN}-\mathrm{HNC}$ and $\mathrm{HNC}-\mathrm{HNC})$ are at least $10 \mathrm{~kJ} \mathrm{~mol}^{-1}$ stronger than the H-bonds with HCN as donor. This behavior is due to the larger positive charge density at the hydrogen in the HNC molecule compared to the HCN. For instance, the calculated (B3LYP/cc-pVDZ) APT atomic charges at the hydrogen are +0.25 and +0.40 in $\mathrm{HCN}$ and $\mathrm{HNC}$, respectively. In addition, the intermolecular charge transfer in the HCN-HNC and HNC-HNC dimers are larger than those in $\mathrm{HCN}-\mathrm{HCN}$ and $\mathrm{HNC}-\mathrm{HCN}$ ones, which can be correlated to the strengthen of the H-bond. ${ }^{14}$ These observations can also be used to explain the differences on the binding energies for the trimeric species. Noteworthy 
that the dispersion and long-range corrections shown in Table 1 lead to overestimation the binding energies. In fact, the results obtained with the BP86 functional without any of these corrections are in very good agreement with the reference $(\mathrm{CCSD}(\mathrm{T}) / \mathrm{CBS})$ data. The trend observed by including the dispersion correction corroborates recent analysis of a set of small H-bonded complexes. 15 In this analysis, the electrostatic contributions are the determining factor in the intermolecular interactions and the inclusion of dispersion corrections in the most density functionals leads large errors. Notice that this situation changes when the BSSE correction is taken in account. However, because the interest is to perform BOMD simulations during which the BSSE correction is not considered, the best compromise (error cancelation) is the BP86/cc-pVDZ method for describing the H-bond energy in dimers. This is quite fortunate because this method does not present a high computational demand and provides results at the $\operatorname{CCSD}(\mathrm{T}) / \mathrm{CBS}$ quality. A quantitative analysis can be ascertained from Table 2, which presents the mean absolute error on the energies for the 12 investigated species: HCN-HCN, HCN-HNC, HNC-HCN, HNCHNC, HCN-HCN-HCN, HCN-HCN-HNC, HCN-HNC$\mathrm{HCN}, \mathrm{HCN}-\mathrm{HNC}-\mathrm{HNC}$, HNC-HCN-HCN, HNC-HCN$\mathrm{HNC}$, HNC-HNC-HCN, and HNC-HNC-HNC, taking the $\operatorname{CCSD}(\mathrm{T}) / \mathrm{CBS}$ results as reference. The qualitative analysis observed for the binding energies of the dimers is quantitatively corroborated.

Table 2. Mean absolute error $\left(\mathrm{kJ} \mathrm{mol}^{-1}\right)$ for the binding energy with respect to $\mathrm{CCSD}(\mathrm{T}) / \mathrm{CBS}$.

\begin{tabular}{|c|c|c|c|}
\hline & cc-pVDZ & cc-pVTZ & cc-pVQZ \\
\hline B3LYP & 3.65 & 2.79 & 4.07 \\
\hline B3LYP-GD3 & 8.16 & 1.72 & 0.49 \\
\hline B3LYP-LR & 7.90 & 0.95 & 0.61 \\
\hline TPSS & 3.18 & 2.25 & 3.02 \\
\hline TPSS-GD3 & 7.69 & 2.37 & 1.59 \\
\hline TPSS-LR & 6.90 & 1.07 & 0.56 \\
\hline BP86 & 1.63 & 4.74 & 5.88 \\
\hline BP86-GD3 & 5.98 & 1.41 & 2.09 \\
\hline BP86-LR & 12.61 & 6.13 & 5.32 \\
\hline PBE & 7.58 & 1.49 & 1.35 \\
\hline PBE-GD3 & 12.08 & 5.62 & 4.57 \\
\hline PBE-LR & 8.67 & 2.26 & 1.42 \\
\hline
\end{tabular}

Namely, the inclusion of dispersion and long-range corrections lead to overestimated results for the smaller basis set (cc-pVDZ). For triple and quadruple-zeta quality basis sets these corrections become relevant and errors decrease, except for the PBE density-functional. In fact, for this functional, the dispersion correction is a problem because it causes significant overestimation even for large basis set. Whereas, the B3LYP results improve with dispersion and long-range corrections for cc-pVXZ X $=\mathrm{T}$ and $\mathrm{Q}$ basis sets. For the smaller basis set, the BP86 functional, without any correction, provided the best results. This very fortunate cancelation of errors allows the application of the BP86/ cc-pVDZ method within BOMD simulations because the inclusion of BSSE and long-range corrections are very troublesome.

An important characteristic of $\mathrm{H}$-bonds is their cooperative enhancement when the number of hydrogen bonds increases. For the trimeric H-bonded complexes (see Table 3), there are cooperativity effects, in addition to the observed influence of the nature of the donor in the H-bond: HCN X HNC. The cooperative energy for the trimeric complexes was obtained by subtracting from their binding energy, the respective energies from the dimeric species that form the H-bonds. In the Table 3, the cooperative energies calculated for all possible trimeric species of $\mathrm{HCN} / \mathrm{HNC}$ are presented. It can be noticed that the cooperative energy increases as the number of HNC molecule increases in the complex. This increase of the cooperative energy is roughly $2 \mathrm{~kJ} \mathrm{~mol}^{-1}$ per unit of $\mathrm{HNC}$ moiety that is a donor in the $\mathrm{H}$-bond. Because the minimum cooperative energy is ca. $5 \mathrm{~kJ} \mathrm{~mol}^{-1}$ (HCN-HCN-HCN) it almost double when the number of HNC donors doubles (HCN-HNC-HNC and HNC-HNC-HNC). Noteworthy that the DFT/cc-pVDZ methods provide similar results for the H-bond cooperative energy and they systematically overestimate this quantity compared to the $\operatorname{CCSD}(\mathrm{T}) / \mathrm{CBS}$ calculations. This is probably due to the basis set effects.

These findings suggest that the liquid mixtures of these $\mathrm{HCN} / \mathrm{HNC}$ molecules should have distinct topological behavior depending upon the mole fraction of each species. For instance, in a 50:50 mixture, the H-bond networked system most likely would prefer to make intercalated bonds of HCN and HNC instead of bonds between molecules of the same type. However, the large differences between the pure $\mathrm{HCN}$ and $\mathrm{HNC}$ networks also suggest that phase separation 
Table 3. Cooperative energies (kJ mol-1) for HCN/HNC trimers calculated with the DFT/cc-pVDZ methods and CCSD(T)/CBS.

\begin{tabular}{|c|c|c|c|c|c|}
\hline Complex & B3LYP & TPSS & BP86 & PBE & CCSD(T)/CBS \\
\hline HCN-HCN-HCN & 5.31 & 5.31 & 5.48 & 5.65 & 4.78 \\
\hline HCN-HCN-HNC & 7.20 & 7.36 & 7.45 & 7.53 & 6.44 \\
\hline HCN-HNC-HCN & 6.86 & 6.90 & 7.07 & 7.15 & 5.96 \\
\hline HCN-HNC-HNC & 9.96 & 10.33 & 10.59 & 10.67 & 8.03 \\
\hline HNC-HCN-HCN & 5.44 & 5.52 & 5.61 & 5.73 & 4.81 \\
\hline HNC-HCN-HNC & 7.91 & 5.44 & 5.27 & 5.19 & 6.94 \\
\hline HNC-HNC-HCN & 7.41 & 7.61 & 7.87 & 8.03 & 6.07 \\
\hline HNC-HNC-HNC & 10.84 & 11.51 & 11.80 & 12.01 & 8.20 \\
\hline
\end{tabular}

at particular thermodynamics situation may occur. In fact, these cooperativity effects can have considerable influence upon the topological structures of these networks in the liquid phase. As a perspective, topological studies of these liquid systems may provide relevant information on how these molecules interact in a complex network and how this network behave upon external perturbations.

\section{Conclusions}

$\mathrm{CCSD}(\mathrm{T}) / \mathrm{CBS} / \mathrm{MP} 2 / 6-311++\mathrm{G}(\mathrm{d}, \mathrm{p}) \quad$ calcu-lations provided a benchmark to ascertain the reliability of density functionals, basis sets, BSSE, dispersion and long-range corrections for determining $\mathrm{H}$-bond energies in dimeric and trimeric species of $\mathrm{HCN} / \mathrm{HNC}$ and the cooperative energies in the trimeric complexes. One of the most reliable and precise method was the BP86/cc-pVDZ without any correction. This is very fortunate because it is the simplest and the least demanding method to perform BOMD simulations of liquid $\mathrm{HCN} / \mathrm{HNC}$ mixtures. In addition, the cooperative effects of the H-bonds may significantly affect the topological behavior of the H-bond networks in these mixtures.

Because the H-bond networks in liquids can be large, we are currently investigating these cooperativity effects within the H-bonds of larger (tetramer and pentamer) $\mathrm{HCN} / \mathrm{HNC}$ complexes with the BP86/cc-pVDZ method.

\section{Acknowledgments}

The authors are grateful for the support given from CAPES, CNPq, FACEPE, FINEP and inct-INAMI.

1. Silva. J. A. B.; Moreira. F. G. B.; dos Santos. V. M. L.; Longo. R. L.,
Phys. Chem. Chem. Phys., 13, 593 (2011).

2. Silva. J. A. B.; Moreira. F. G. B.; dos Santos. V. M. L.; Longo. R. L., Phys. Chem. Chem. Phys., 13, 6452 (2011).

3. Silva. J. A. B.; Moreira. F. G. B.; dos Santos. V. M. L.; Longo. R. L., Phys. Chem. Chem. Phys., 16, 19479 (2014).

4. Mamajanov. I.; Herzfeld. J.; J. Chem. Phys., 130, 134503 (2009).

5. Matthews. C. N., Orig. Life Evol. Biosphere, 21, 421 (1991).

6. Martiniano. H. F. M. C.; Cabral. B. J. C.; Chem. Phys. Lett., 555, 119 (2013).

7. Del Bene, J. E.; Alkorta. I.; Elguero. J., J. Phys. Chem. A., 114, 8463 (2010).

8. Provasi. P. F.; et al., J. Phys. Chem. A., 109, 6555 (2005).

9. Adrian-Scotto. M.; Vasilescu. D., J. Mol. Struct. (Theochem), 803, 45 (2007).

10. Truhlar. D. G., Chem. Phys. Lett., 294, 45 (1998).

11. Huh. S. B.; Lee. J. S., J. Chem. Phys., 118, 73035 (2003).

12. Mettee. H. D., J. Phys. Chem., 77, 14 (1973).

13. Giauque. W. F.; Ruehrwein. R. A., J. Am. Chem. Soc., 61, 2626 (1939).

14. Araújo. R. C. M. U.; Ramos M. N.; J. Braz. Chem. Soc., 9, 499 (1998).

15. 15Boese, D. A.; ChemPhysChem, 16, 978 (2015).

\section{Paulo McMiller C. de Oliveira $^{a^{*}}$, Juliana A. B. Silva ${ }^{b}$ \& Ricardo L. Longo ${ }^{a}$}

\footnotetext{
a Departamento de Química Fundamental, Universidade Federal de Pernambuco, 50740-540, Recife, PE, Brazil,

${ }^{\mathrm{b}}$ Centro Acadêmico do Agreste, Universidade Federal de Pernambuco, 55002-970, Caruaru, PE, Brazil

*E-mail: mcmiller@live.nl
} 